\title{
Design and control of different pressure thermally coupled reactive distillation for methyl acetate hydrolysis
}

\author{
Lumin Li, ${ }^{\text {a }}$ Lanyi Sun, ${ }^{,}$a Jun Wang, ${ }^{\mathrm{b}}$ Jian Zhai, ${ }^{\mathrm{a}}$ Yuliang, Liu ${ }^{\mathrm{a}}$, Wang Zhong ${ }^{\mathrm{a}}$ and Yuanyu Tian ${ }^{\mathrm{a}}$ \\ ${ }^{a}$ State Key Laboratory of Heavy Oil Processing, China University of Petroleum, Qingdao 266580, China \\ ${ }^{\mathrm{b}}$ China Petroleum Engineering Construction Corporation Dalian Design Company, Dalian 116011, China
}

Corresponding Author: Lanyi Sun. Tel.: +86 13854208340. Fax: +86 053286981787.

E-mail address: sunlanyi@upc.edu.cn. 


\section{SUPPORTING INFORMATION}

1. The equipment is sized as follows:

(1) The heat transfer area $(A)$ in the condenser and reboiler

$A\left[m^{2}\right]=\frac{Q}{U \cdot \Delta T}$

Here, $\Delta T(\mathrm{~K})$ is the logarithmic mean temperature difference; $Q(\mathrm{~kW})$ is the heat duty; $U\left(\mathrm{~kW} /\left(\mathrm{K} \cdot \mathrm{m}^{2}\right)\right)$ is the heat transfer coefficient.

(2) The height of the column

$L_{c}[m]=\left(N_{\text {actual }}-1\right) \times 0.6096+6$

Here, $0.6096 \mathrm{~m}$ is the tray spacing and $6 \mathrm{~m}$ is the disengagement.

2. The capital cost is calculated as follows:

(1) Installed column cost

Installed column shells cost $[\$]=\frac{M \& S}{280} \times 937.636 \times D^{1.066} \times L_{c}^{0.802} \times\left(2.18+F_{c}\right)$

Here, $F_{c}=F_{m} \times F_{p} ; F_{m}$ and $F_{p}$ are the construction material and pressure range, respectively. The ratio of Marshall \& Swift index $(M \& S)$ is specified at 1468.2. $\quad F_{m}$ is set at 1 and $F_{p}$ is set at 1.

Installed column trays cost $[\$]=\frac{M \& S}{280} \times 97.243 \times D^{1.55} \times h_{T} \times F_{c}$

Here, $F_{c}=F_{s}+F_{t}+F_{m}$, and $h_{T}(\mathrm{~m})=\left(N_{\text {actual }}-1\right) \times 0.6096$.

(2) Installed heat exchanger cost

Installed heat exchanger cost $[\$]=\frac{M \& S}{280} \times 474.668 \times A^{0.65} \times\left(2.29+F_{c}\right)$

Here, $F_{c}=\left(F_{d}+F_{p}\right) \times F_{m}$. For reboiler, $F_{c}=(1.35+0) \times 1=1.35$.

(3) Installed compressor cost

Installed compressor cost $[\$]=\frac{M \& S}{280} \times 666.1 \times P^{0.82} F_{c}$ 
Here, $F_{c}$ varies with design types as follows: 1.00 for centrifugal (motor), 1.07 for reciprocating (steam), 1.15 for centrifugal (turbine), and 1.29 and 1.82 for reciprocating (motor and gas engine).

3. The operating cost is calculated as follows:

(1) Steam cost

Steam cost $[\$ / \mathrm{y}]=C_{s} \times \frac{Q_{R}}{\lambda_{V}} \times 8000 \times 3600$

Here, $C_{s}(\$ / \mathrm{kg})$ is the saturated steam price; $\lambda_{V}(\mathrm{~kJ} / \mathrm{kg})$ is the latent heat of the steam which depends on the bottom temperature; $Q_{R}(\mathrm{~kW})$ is the reboiler heat duty.

(2) Cooling water cost

Cooling water cost $[\$ / y]=\frac{0.03}{3.785} \times \frac{Q_{C}}{\Delta T \cdot C_{p} \cdot 1000} \times 8000 \times 3600$

Here, $Q_{C}(\mathrm{~kW})$ is the condensor heat duty; $C_{p}(\mathrm{~kJ} /(\mathrm{kg} \cdot \mathrm{K}))$ is the specific heat capacity of the cooling water; $\Delta T(\mathrm{~K})$

is the temperature difference between the outlet and inlet of the cooling water.

(3) Catalyst cost (assuming a catalyst life of 3 months)

Catalyst cost $[\$]=$ catalyst loading $[\mathrm{kg}] \times 7.7162 \frac{\$}{\mathrm{~kg}}$ 